\title{
Unpredictable Changes: Different Effects of Derailment on Well-Being Between North American and East Asian Samples
}

\author{
Yuta Chishima' ${ }^{1}$ (D) Masato Nagamine ${ }^{2}$
}

Accepted: 18 February 2021 / Published online: 8 March 2021

(C) The Author(s), under exclusive licence to Springer Nature B.V. 2021

\begin{abstract}
Some individuals experience the feeling that they have become a person they had not anticipated. The life path they had expected to take is not consonant with the one they are taking in reality. This perception of "off-course" in identity and self-direction is referred to as derailment. Although previous studies have postulated and demonstrated that derailment causes a low level of well-being, no studies have examined its existence and effect across cultures. We hypothesized that East Asians (Japanese) are less vulnerable to feeling derailed than North Americans (Canadians/Americans), and that those Japanese who feel derailed do not necessarily experience long-term damage to their well-being. Two correlational studies and one longitudinal study with a one-year interval supported these hypotheses and also demonstrated metric invariance of the Derailment Scale between countries. We discuss that these findings may be explained by East Asian's dialectical thinking, in which the perception of one's life direction is flexible.
\end{abstract}

Keywords Derailment $\cdot$ Well-being $\cdot$ Culture $\cdot$ Dialecticism $\cdot$ Identity

\section{Introduction}

Throughout one's lifespan, some individuals experience changes in who they are and which directions they choose in life; that is, they occasionally notice that they have become a person whom they had not imagined. The life course they had expected to take is not always compatible with the one they are currently taking. This perception of off course in identity and self-direction is referred to as "derailment," which is defined as the perception that who one is has changed over time in constitution and course (Burrow et al., 2020).

Yuta Chishima

chishima.yuta.fw@u.tsukuba.ac.jp

1 Faculty of Human Sciences, University of Tsukuba, Tsukuba 3058572, Japan

2 Graduate School of Comprehensive Human Sciences, University of Tsukuba, Tsukuba, Japan 


\subsection{The Concept of Derailment}

Burrow et al. (2020) theorized a sense of derailment is comprised of temporal discordance in one's sense of self and direction as follows. First, it is required that derailed individuals believe that their important past identity has been extinguished or replaced by a different one. They feel they have a discrepancy between who they are and who they used to be. Second, individuals who experience derailment recognize that they cannot follow the past life direction they used to take. They sense a substantial transformation in the direction of their life, perceiving they cannot pursue their dream or plan that they had in the past. Third, highly derailed individuals are likely to note difficulties in imagining how their past self has a deep connection to their present self. In other words, derailment stands for identity-relevant loss of the sense of how who they were is meaningfully relevant to who they currently are. To summarize, this novel construct captures a sense of losing or changing identities and life paths individuals had in the past, and, more importantly, failing to draw a meaningful connection between temporally distinct identities. Integrating these components into a concept is the unique characteristics of derailment, which makes derailment distinct from conceptually similar constructs, such as narrative identity (e.g., McAdams \& McLean, 2013), self-concept clarity (e.g., Campbell et al., 1996), temporal self-comparison (e.g., Keyes \& Ryff, 2000), or self-continuity (e.g., Sedikides et al., 2015).

It is also noteworthy that derailment represents "subjective" (not objective) change in identity and self-direction. Imagine two different men with advanced knowledge of computers who had to change their occupation from software engineer to farmer. One man thinks the transition in his career path is meaningfully connected and tries applying his skills to his new farming career, whereas the other man feels no connection between who he was as a software engineer and who he currently is as a farmer. He views himself as completely different from who he was; consequently, there is the sense of being off-course in his career path. The description of the first man is someone who would be regarded as less derailed, while the second man would be regarded as highly derailed. Although they experienced the same career transition, subjective perception of the change differs between the two men, leaving one feeling more derailed than the other. Although measuring how much individuals have changed over time is a typical methodological strategy (e.g., Harter et al., 1997; Meeus et al., 1999; Roberts et al., 2006; Specht et al., 2011), the subjective perception of change often mismatches the objective change (Lachman et al., 2008; Robins et al., 2005; Wilson \& Ross, 2001). Therefore, subjective perception of change in identity or life direction could provide informative findings that traditional measurement scales have not been able to capture.

\subsection{Derailment and Well-Being}

As characterized by the research in the area of identity formation, perceiving instability and discontinuity in one's self can be damaging to one's well-being (e.g., Ball \& Chandler, 1989; Erikson, 1968; Keyes, 2000; Keyes \& Ryff, 2000). Importantly, previous studies have indicated that participants who reported a greater magnitude of change in their lives experienced an attenuation in their psychological functioning, regardless of whether participants reported they had changed for the better or worse (Burrow et al., 2014, 2020; Church et al., 2014; Haas \& van Dellen, 2020; Keyes \& Ryff, 2000). Indeed, studies using the Derailment Scale developed by Burrow et al. (2020) have found evidence that a highly 
derailed individual is likely to report a low level of well-being, such as a low level of life satisfaction and positive affect, and high level of depression (Burrow et al., 2020; Ratner et al., 2019, 2020). Specifically, Burrow and colleagues reported in a series of studies that derailment was positively associated with the above-mentioned negative outcomes even after accounting for demographic characteristics (age and gender), identity-related variables (identity formation, self-concept clarity, and self-continuity), the Big-Five personality domains, and the number of status-changing life events experienced in the past.

A common finding of longitudinal studies is that a high level of derailment predicted increased depression over time. Burrow et al. (2020) revealed that derailment had a longterm influence on fostering depressive symptoms using an 18 month longitudinal survey. This effect still remained after controlling for personality. Using the same measure as did Burrow et al. (2020), Ratner et al. (2019) conducted a multi-wave study over a period of one year and found similar results with each two-month interval.

\subsection{Cultural Differences in the Impact of Derailment on Well-Being}

Although cultural differences in the impact of derailment on well-being is currently unknown, there are some previous studies about temporal instability of the self-concept across cultures (Chopik \& Kitayama, 2018; Church et al., 2012, 2014; Haas \& van Dellen, 2020), which could contribute to generating hypotheses about how culture plays a role as a buffer in the relationship between derailment and well-being. For instance, Church et al. (2014) revealed that objective short-term (one month) temporal instability of the selfconcept had stronger and more reliable negative correlations with subjective well-being in Western cultures (e.g., American), compared to Eastern cultures (e.g., Japanese). More recent research has also replicated this finding with regard to long-term instability even after a nine year interval, using Japanese and American longitudinal datasets (Haas and van Dellen, 2020). Drawing on these previous studies, it appears that the association of derailment with well-being can be moderated by culture, indicating East Asians are relatively more resilient to derailment.

Given prior research findings, the question that arises is why does the effect of experiencing temporal inconsistency of the self differ between cultures? It can be presumed that East Asians may be less vulnerable to feeling derailed because they hold a unique lay theory called "dialecticism" (Boucher, 2011; Heine, 2001; Nisbett et al., 2001; Peng \& Nisbett, 1999; Spencer-Rodgers et al., 2009, 2010), which stems from Chinese religious, philosophical, and epistemological traditions that have been widespread over East Asia. This traditional way of thinking is composed of three concepts: (a) tolerance for contradiction, referring to both sides of an apparent contradiction will include some piece of the truth; (b) the expectation of change, characterized as the perception that everything is unpredictable, dynamic, and in constant flux; and (c) cognitive holism, in which everything in the universe is interconnected and mutually dependent.

Dialecticism, or dialectical thinking, has been exemplified in empirical studies about cultural differences in perception of change. Ji et al. (2001) found when participants were asked to indicate what the next trend would be in choosing from graphs with particular trends, Chinese, compared to Americans, were more likely to reflect the belief that the direction of the trend would change. Additionally, they found that Chinese participants were more likely than Americans to pick out non-linear or parabolic trends of happiness across the lifespan when many different kinds of happiness trends were displayed. It is possible that the flexible and compromising perception of one's life direction, which is as 
an aspect of East Asian culture, could buffer the detrimental effects of derailment on wellbeing. Conversely, the essential mechanism behind the harmful effects of derailment in North Americans could be due to the fixed linear perception of one's life trajectory, which can contribute to imagining that one's derailed life path will continue without recovering the self one used to be.

\subsection{The Current Study}

The aim of the current study was to examine the differences in the impact of derailment on well-being between East Asians and North Americans. We posited that derailment in East Asians would have a weaker negative correlation with well-being than in North Americans (Hypothesis 1). Additionally, we expected that East Asians who feel derailed would not necessarily experience long-term damage to their well-being (Hypothesis 2). To test the first hypothesis we conducted two studies. In Study 1, we used data from Canadian and Japanese samples, and in Study 2, we used data from American and Japanese samples. To test the second hypothesis, Study 3 used longitudinal data from a Japanese sample with a one year interval. Although the sample included only Japanese participants, we could compare our results to the results of the American sample in Burrow et al. (2020) in which they conducted a similar longitudinal study with a one and a half year interval.

To assess participants' well-being, we adopted different scales as measures of the construct. Well-being can be classified as either hedonic or eudaimonic (c.f., Huta, 2016; Ryan $\&$ Deci, 2001). We focused only on hedonic well-being, because most of the research about derailment has examined hedonic well-being and we attempted to replicate and extend previous research. First, we assessed present life satisfaction, future life satisfaction, and positive affect given that the most common approach to studying subjective well-being is to distinguish it into cognitive and affective aspects (c.f., Diener, 1984). Life satisfaction is considered a cognitive aspect of well-being because it is based on evaluative beliefs about one's life, whereas positive affect reflects the affective state of one's life (Schimmack, 2008). Including an assessment of future life satisfaction is informative because it can explain whether derailment has an influence on one's anticipated well-being in the future. Second, we took into account cultural aspects of positive affect. To do so we included not only high-arousal positive affect (e.g., fun), but also low-arousal positive affect (e.g., relaxed), which is highly valued in East Asian culture compared to North American culture (Tsai et al., 2006). Lastly, in accord with previous studies (Burrow et al., 2020; Ratner et al., 2019, 2020), we also examined the effect of derailment on depressive symptoms. Focusing on different aspects of well-being would heighten the generalizability of our results.

In advance of examining the association between derailment and well-being, we explored the factor structure of the scale used to assess derailment across all three studies. We conducted confirmatory factor analysis (CFA) with invariance testing to evaluate whether the scale was conceptually similar to respondents from both cultures. The test of measurement invariance across cultures is highly recommended in cross-cultural studies (Boer et al., 2018). In the CFA, we used the following criteria to evaluate the model fit: CFI and TLI values greater than 0.90 indicate acceptable fit, and values of these indices greater than 0.95 indicate close fit; SRMR and RMSEA values less than 0.08 indicate acceptable fit, and values below 0.05 indicate close fit (Marsh et al., 2004). The Bayesian information criterion was used when we compared models to choose the better one. 


\subsection{Data Analyses}

Total scores on the scales were calculated (negatively worded items were reverse scored) and averaged for each scale. Data were analyzed using SPSS ver. 25 and Mplus ver. 8.3.

\section{Study 1}

In Study 1, we examined whether there would be cultural differences in the relationships between derailment and present and future life satisfaction, focusing on Canadian undergraduate students as the North American sample and Japanese undergraduate students as the East Asian sample.

\subsection{Method}

\subsubsection{Participants}

The Canadian sample consisted of 890 students from a Canadian university. We selected only White/Caucasian ethnicities. The Japanese sample consisted of 940 students from various universities in Japan. We excluded individuals who did not have Canadian or Japanese citizenship, came from international universities, were over 25 years of age, did not pass an attention check, and did not finish the questionnaire. The final sample consisted of 839 Canadian participants $\left(M_{\text {age }}=18.79\right.$ years, $S D=1.34$, range 16-25, four did not report age; $81.5 \%$ were women) and 900 Japanese participants $\left(M_{\text {age }}=20.74\right.$ years, $S D=1.54$, range $18-25 ; 40.2 \%$ were women $)$. A posteriori power analysis showed that this sample size was large enough for detecting a small effect size $\left(f^{2}=0.02\right.$ resulted in $\left.1-\beta=0.99\right)$ when we ran the following moderation analyses with covariates.

\subsubsection{Procedures}

The Canadian sample was recruited through the psychology department subject pool at a Canadian university in which students received course credit for participation. The Japanese sample was recruited using two sources: an online platform called Lancers, which is a crowdsourcing website; and an online survey company called Macromill that has a large pool of research participants in Japan. We merged the two Japanese samples after checking that there were no large differences in age, gender, and scores on the measures. They received compensation (e.g., 50 Japanese yen) after participation. Participants completed online questionnaires anonymously. They consented to participation in the study before responding to the questionnaires. They were informed that participation was voluntary, and that there would be no consequence for omitting responses or declining to participate. This study was approved by the research ethics committees of Kyoto University, University of Tsukuba, and Wilfrid Laurier University. 


\subsubsection{Measures}

2.1.3.1 Derailment We assessed derailment using the 6-item short form of the Derailment Scale that was developed by Burrow et al. (2020). Items are rated on a 5-point Likert scale ranging from 1 ("strongly disagree") to 5 ("strongly agree"). Example items include, "I did not anticipate becoming the person that I currently am" and "I feel like I've become a different type of person over time." The original measure consists of 10 items; however, the 6-item scale was adopted for three reasons. First, Burrow et al. (2020) demonstrated that the 10 -item scale consisted of two factors that could be distinguished on the basis of whether participants reflect on differences or similarities between current and past selves. Second, they compared results using the 10-item version with the 6-item version and reported the pattern of results were not different, suggesting researchers can use either version. Third, the model fit indices of the 10-item scale they reported in a series of studies did not satisfy criteria. For instance, the model indices in one study were $\mathrm{CFI}=0.68$ and $\mathrm{RMSEA}=0.15$, while in another study the indices were CFI $=0.63$ and RMSEA $=0.17$ (Burrow et al., 2020). Even after they put covariances with residuals to modify the model, the indices did not meet criteria (e.g., the CFI did not go over 0.90 ). This drawback could be troublesome especially when comparing models across cultures. Albeit Burrow and colleagues did not report the fit indices of the 6-item scale, model fit is assumed to be better because a smaller number of items generally increases fit indices (Ding et al., 1995). They provided evidence in support of the reliability and validity of the scale for both undergraduates and adults. In the current study, Cronbach's $\alpha=0.79$ in the Canadian sample and $\alpha=0.72$ in the Japanese sample.

The original English version of the Derailment Scale was translated into Japanese using a translation and back-translation process (Brislin, 1986). First, three professional translators translated the scale into Japanese without discussing or checking the others' translation. Second, the two native Japanese authors of this manuscript chose the best translation of each item, correcting the wording slightly to read more naturally, as necessary. Third, different professionals translated those items into English. Finally, the researcher who developed the original scale certified that the original items and the back-translated items had the same meaning.

2.1.3.2 Life Satisfaction We assessed present and future life satisfaction using two subscales from the Adolescent Time Attitude Scale (ATAS; Worrell et al., 2013): the Present Positive and Future Positive subscales. The main reason for choosing the ATAS was that it can assess both present and future life satisfaction in the same scale, and the reliability and validity of both the English (Worrell et al., 2013) and Japanese (Chishima et al., 2019) versions have been confirmed. This scale was originally developed to assess adolescents' time attitudes; however, it is also applicable to adult populations (Mello et al., 2016). Each subscale consists of five items. Example items are, "I am content with the present" for present life satisfaction and, "My future makes me happy" for future life satisfaction. The items are rated on a 5-point Likert scale ranging from 1 ("strongly disagree") to 5 ("strongly agree"). In the current study, Cronbach's alphas for the Present Positive subscale were 0.94 in the Canadian sample and 0.89 in the Japanese sample. Cronbach's alphas for the Future Positive subscale were 0.96 in the Canadian sample and 0.93 in the Japanese sample. 


\subsection{Results}

\subsubsection{Measurement Tests of the Derailment Scale}

We performed principal component analysis (PCA) to identify the factors of the Derailment Scale in the Japanese and Canadian samples, following Burrow et al. (2020) procedure. The scree plot indicated a sharp break between the first and the second factor in both samples (Eigenvalues were 3.016, 0.862, 0.761, 0.570 . for the Canadian sample; $2.629,0.990,0.785,0.705 \ldots$ for the Japanese sample), which confirmed the unidimentionality of the scale across samples. As can be seen in Table 1, all factor loadings were high except for Item 2 ("I do not feel very connected to who I was in the past") in the Japanese sample.

Next, we conducted CFA to examine the model structure of the Derailment Scale, showing acceptable fit in each sample although RMSEA was over 0.10 in the Canadian sample. We examined three models to evaluate measurement invariance in the two samples: configural invariance, metric invariance, and scalar invariance. The configural model has factor loadings, intercepts, and residual variances free across the samples. The metric model has factor loadings constrained to be equal across the samples, while intercepts and residual variances are free across the samples. This level of invariance allows for the comparison of bivariate relations, such as factor correlations and correlations to covariates between countries. The scalar model has factor loadings and intercepts constrained to be equal across the samples, while residual variances are free across the samples (Byrne, 2013). This level of invariance allows for the unbiased comparisons of factor means. As can be seen in Table 2, the configural and metric invariance models had almost acceptable fit, and the Bayesian information criterion (BIC) coefficients indicate the metric invariance model was better than the other models. Using the same procedure employed in the Burrow et al. (2020) studies, we put covariances with residuals between Item 3 and Item 6 and between Item 4 and Item 5 to examine whether a better model fit would be observed. Results showed that the model indices were improved after adding the covariances, the BIC indicating the modified metric invariance model was the best fit $(\mathrm{CFI}=0.973$, TLI $=0.957$, $\mathrm{SRMR}=0.050$, RMSEA $=0.066$ ). The factor loadings for the model in both samples are displayed in Table 1.

\subsubsection{Descriptive Statistics and Correlations}

Descriptive statistics and correlations among the variables are presented in Table 3. Derailment scores significantly differed by country, which were higher in the Canadian than in the Japanese sample $(t=6.08, d f=1,737, p<0.001, d=0.29)$. Likewise, scores for present and future life satisfaction (LS) significantly differed by country, which were higher in the Canadian sample $(t=13.73, d f=1,737, p<0.001, d=0.66$ for present LS; $t=17.53, d f=1,737, p<0.001, d=0.84$ for future LS). There were significant negative correlations between derailment and present and future life satisfaction in the Canadian sample $(r=-0.27, p<0.001$ for present LS; $r=-0.18, p<0.001$ for future LS), which is consistent with previous research (Burrow et al., 2020), whereas the correlations in the Japanese sample were not significant $(r=-0.02$, n.s. for present LS; $r=0.00$, n.s. for future LS). 


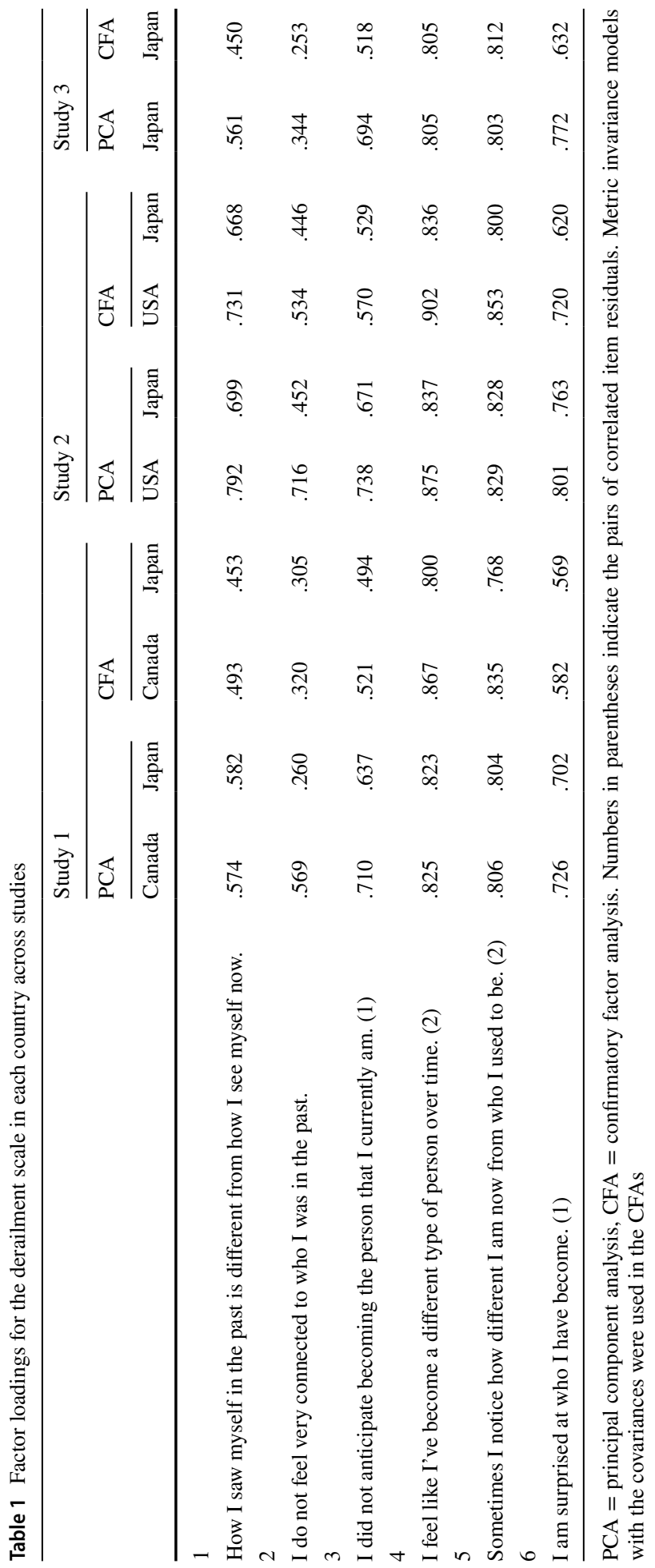


Table 2 Fit indices for the derailment scores derived from confirmatory factor analyses across studies

\begin{tabular}{|c|c|c|c|c|c|c|c|c|c|}
\hline Model & $\chi^{2}$ & $d f$ & $\chi^{2} / d f$ & CFI & TLI & SRMR & RMSEA & $90 \% C I$ & $\mathrm{BIC}$ \\
\hline \multicolumn{10}{|l|}{ Study 1} \\
\hline Only Canada & $89.18^{*}$ & 9 & 9.91 & .945 & .909 & .039 & .103 & [.084 .123] & $13,077.82$ \\
\hline Only Japan & $39.14 *$ & 9 & 4.35 & .973 & .956 & .028 & .061 & [.042 .081] & $15,076.65$ \\
\hline Only Canada ${ }^{a}$ & $31.35^{*}$ & 7 & 4.48 & .983 & .964 & .025 & .064 & [.042 .088] & $13,033.45$ \\
\hline Only Japan ${ }^{\mathrm{a}}$ & $25.74 *$ & 7 & 3.68 & .983 & .965 & .024 & .055 & ].033 .078] & $15,076.84$ \\
\hline Configural & $128.32 *$ & 18 & 7.13 & .958 & .929 & .034 & .084 & [.071 .098 ] & $28,179.44$ \\
\hline Metric & $164.99 *$ & 23 & 7.17 & .945 & .929 & .059 & .084 & [.072.097] & $28,178.80$ \\
\hline Scalar & $412.82^{*}$ & 28 & 14.74 & .852 & .841 & .089 & .126 & [.115 .137] & $28,389.32$ \\
\hline Configural $^{\mathrm{a}}$ & $57.09^{*}$ & 14 & 4.08 & .983 & .964 & .024 & .059 & [.044 .076] & $28,138.05$ \\
\hline Metric $^{\mathrm{a}}$ & $90.12 *$ & 19 & 4.74 & .973 & .957 & .050 & .066 & [.052.080] & $28,133.78$ \\
\hline Scalar $^{\mathrm{a}}$ & $326.08^{*}$ & 24 & 13.59 & .884 & .855 & .081 & .120 & [.109 .132] & $28,332.42$ \\
\hline \multicolumn{10}{|l|}{ Study 2} \\
\hline Only USA & $72.58 *$ & 9 & 8.06 & .951 & .918 & .039 & .129 & [.102 .157] & 6937.96 \\
\hline Only Japan & $84.50 *$ & 9 & 9.39 & .927 & .879 & .042 & .126 & [.103 .152] & 8160.71 \\
\hline Only Canada ${ }^{a}$ & $49.81 *$ & 7 & 7.12 & .967 & .929 & .034 & .120 & [.090 .152] & 6927.31 \\
\hline Only Japan ${ }^{\mathrm{a}}$ & $28.19 *$ & 7 & 4.03 & .980 & .956 & .023 & .076 & {$[.048 .106]$} & 8116.93 \\
\hline Configural & $157.08 *$ & 18 & 8.73 & .940 & .901 & .041 & .127 & [.109.146] & $15,123.82$ \\
\hline Metric & $180.64 *$ & 23 & 7.85 & .932 & .912 & .068 & .120 & [.104 .137] & $15,113.09$ \\
\hline Scalar & $314.25^{*}$ & 28 & 11.22 & .877 & .868 & .095 & .147 & {$[.132 .161]$} & $15,212.40$ \\
\hline Configural $^{\mathrm{a}}$ & $78.00 *$ & 14 & 5.57 & .973 & .941 & .028 & .098 & {$[.077 .120]$} & $15,072.17$ \\
\hline Metric $^{\mathrm{a}}$ & $107.84 *$ & 19 & 5.68 & .962 & .940 & .059 & .099 & [.081 .118] & $15,067.71$ \\
\hline Scalar $^{\mathrm{a}}$ & $237.28 *$ & 24 & 9.89 & .909 & .886 & .090 & .137 & [.121 .153] & $15,162.87$ \\
\hline \multicolumn{10}{|l|}{ Study 3} \\
\hline Only Japan & $68.07 *$ & 9 & 7.56 & .950 & .916 & .033 & .091 & [.071.112] & $12,159.96$ \\
\hline Only Japan ${ }^{\mathrm{a}}$ & $26.92 *$ & 7 & 3.85 & .983 & .964 & .021 & .060 & [.037 .085] & $12,132.16$ \\
\hline
\end{tabular}

$\mathrm{CFI}=$ robust comparative fit index, TLI $=$ Tucker-Lewis index, SRMR $=$ standardized root mean square residual, $\mathrm{RMSEA}=$ robust root mean square error of approximation; $\mathrm{CI}=$ confidence interval, $\mathrm{BIC}$ $=$ Bayesian information criterion

a These models include the covariances with residuals between Items 3 and 6, and between Items 4 and 5 $* p<.001$

\subsubsection{Moderation Analyses}

To examine whether the correlation coefficients statistically differed between the two samples, we conducted a series of hierarchical multiple regression analyses examining the interaction between country of the participants and derailment. The dependent variables were present and future life satisfaction. We used Hayes' (2013) PROCESS Macro to conduct the analyses. In the first step, the countries and derailment were included. The second step added the interaction variables (country $\times$ derailment). Age and gender were added as covariates to control for their effect on the dependent variables. The $R$-square change for both present and future life satisfaction were significant $\left(\Delta R^{2}=0.01, p<0.000\right.$ for present LS; $\Delta R^{2}=0.01, p=0.001$ for future LS). As shown in Fig. 1, simple slopes tests (Aiken $\&$ West, 1991) showed that in the Canadian sample derailment inhibited present $(\beta=-$ $0.23, p<0.001)$ and future life satisfaction $(\beta=-0.15, p<0.001)$, whereas in the Japanese 
Table 3 Descriptive statistics and correlation matrix in studies 1 and 2

\begin{tabular}{|c|c|c|c|c|c|c|c|}
\hline \multirow[b]{2}{*}{ Study 1} & \multicolumn{2}{|c|}{ Canada } & \multicolumn{2}{|l|}{ Japan } & \multirow[b]{2}{*}{1} & \multirow[b]{2}{*}{2} & \multirow[b]{2}{*}{3} \\
\hline & $M$ & $S D$ & $M$ & $S D$ & & & \\
\hline 1. Derailment & 3.45 & 0.71 & 3.24 & 0.69 & - & $-.27 * * *$ & $-.18 * * *$ \\
\hline 2. Present LS & 3.82 & 0.76 & 3.27 & 0.88 & -.02 & - & $.56 * * *$ \\
\hline \multirow[t]{2}{*}{ 3. Future LS } & 4.03 & 0.87 & 3.23 & 1.03 & .00 & $.54 * * *$ & - \\
\hline & \multicolumn{2}{|l|}{ USA } & \multicolumn{2}{|l|}{ Japan } & & & \\
\hline Study 2 & $M$ & $S D$ & $M$ & $S D$ & 1 & 2 & 3 \\
\hline 1. Derailment & 3.10 & 0.93 & 3.26 & 0.72 & - & $-.27 * * *$ & $-.21 * * *$ \\
\hline 2. Present LS & 5.98 & 1.97 & 4.98 & 2.23 & -.08 & - & $.74 * * *$ \\
\hline 3. Future LS & 6.92 & 2.13 & 5.72 & 2.52 & $.09 *$ & $.64 * * *$ & - \\
\hline
\end{tabular}

LS = life satisfaction. Correlations above the diagonal are for the Canadian/American sample and below are for the Japanese sample

$* p<.05 ; * * * p<.001$
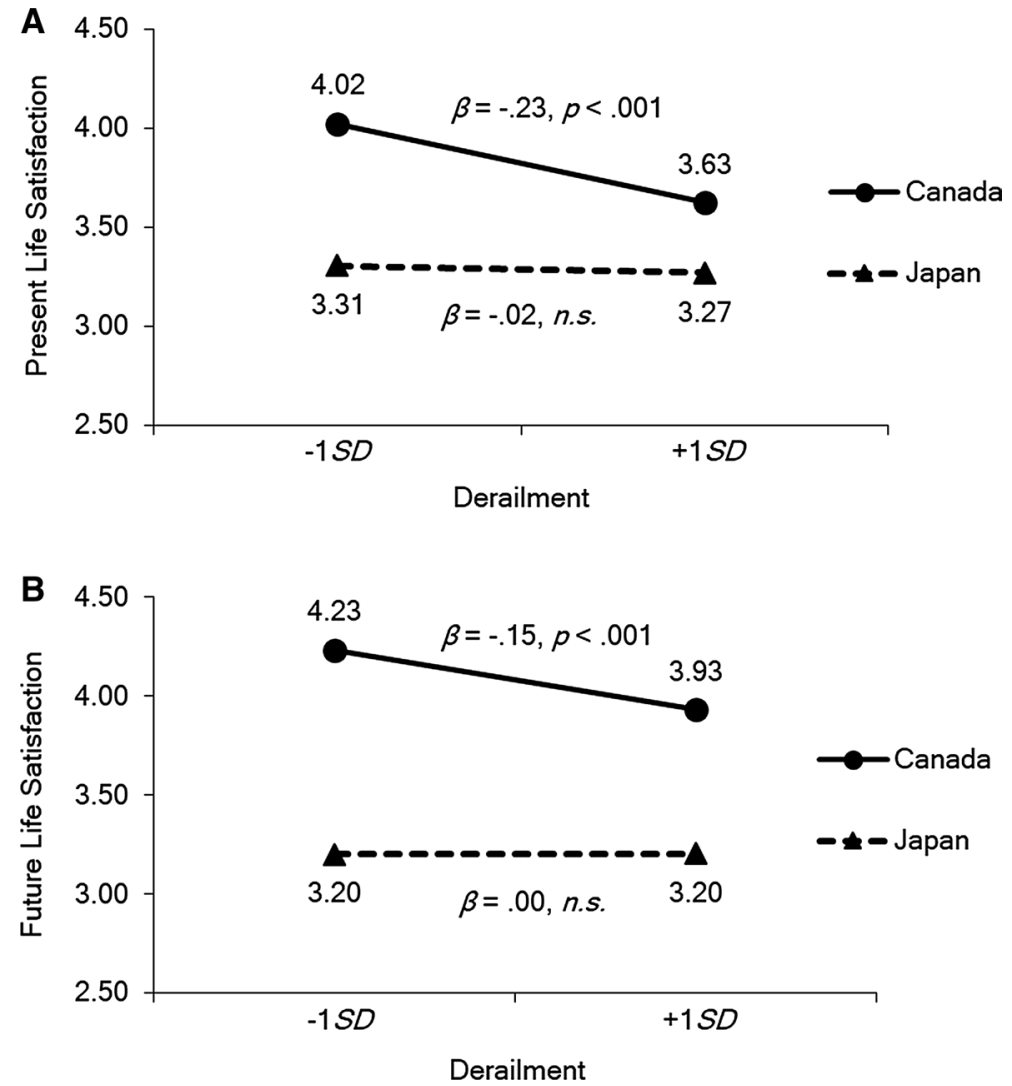

Fig. 1 Interaction Between Countries and Derailment in Study 1 
sample derailment was not significantly related to present $(\beta=-0.02, p=0.510)$ or future life satisfaction $(\beta=0.00, p=0.982)$.

\section{Study 2}

Study 1 showed that derailment was negatively associated with both present and future life satisfaction in the Canadian sample, whereas derailment was not significantly related to life satisfaction in the Japanese sample. In Study 2, we attempted to replicate and expand these results using different measures of life satisfaction and with adult samples in the United States and Japan.

\subsection{Method}

\subsubsection{Participants}

The original sample consisted of 451 Americans and 552 Japanese adults. We selected only White/Caucasian Americans. We excluded individuals who were under 20 years of age, did not currently live in the USA or Japan, did not indicate their first language was English or Japanese, did not pass an attention check, and did not finish the questionnaire. The final sample consisted of 427 American adults $\left(M_{\mathrm{age}}=40.21\right.$ years, $S D=11.05$, range $21-72 ; 49.4 \%$ were women $)$ and 525 Japanese adults $\left(M_{\text {age }}=39.55\right.$ years, $S D=9.69$, range $20-70 ; 53.0 \%$ were women). A posteriori power analysis showed that this sample size was large enough for detecting a small effect size $\left(f^{2}=0.02\right.$ resulted in $\left.1-\beta=0.99\right)$ when we ran the following moderation analyses with covariates Employment status in both samples was as follows (in the order of Americans/Japanese): student $=1.2 / 2.9 \%$, full-time employee $=75.9 / 37.1 \%$, part-time employee $=9.4 / 13.3 \%$, housewife $/$ househusband $=3.7 / 24.8 \%$, unemployed $=3.5 / 9.7 \%$, and other $=6.3 / 12.2 \%$.

\subsubsection{Procedures}

The American sample was recruited by Amazon's Mechanical Turk, which is a commonly used crowdsourcing website. Participants received 2.00 US dollars as compensation. The Japanese sample was recruited using Lancers, as in Study 1. Participants received 200 Japanese yen as compensation. As in Study 1, participants completed online questionnaires anonymously.They consented to participation in the study before responding to the questionnaires. They were informed that participation was voluntary, and that there would be no consequence for omitting responses or declining to participate. This study was approved by the research ethics committee of Kyoto University.

\subsubsection{Measures}

3.1.3.1 Derailment The same short version of the Derailment Scale (Burrow et al., 2020) that was used in Study 1 was used in Study 2. Cronbach's alpha was 0.88 for the American sample and 0.81 for the Japanese sample. 
3.1.3.2 Life Satisfaction We assessed present and future life satisfaction differently than in Study 1 to test the generalizability of the results. The items in Study 2 have been used in national surveys, in particular the Midlife in the United States (MIDUS) and the Midlife in Japan (MIDJA) longitudinal studies. For present life satisfaction, participants were asked, "How would you rate your life overall these days?" For future life satisfaction, they were asked, "Looking ahead 10 years into the future, what do you expect your life overall will be like at that time?" Participants responded to each question using an 11-point Likert scale ranging from 0 ("worst") to 10 ("best"). The future life satisfaction in Study 1 assessed future in general without referring to a particular time span, whereas the one in Study 2 used "10 years later" as a reference for future so that participants have a clear image of how far the future is.

\subsection{Results}

\subsubsection{Measurement Tests of the Derailment Scale}

Following the same method in Study 1, we performed PCA, which confirmed the unidimentionality of the scale across the two samples. Additionally, as can be seen in Table 2, the CFA indicated the modified metric invariance model with covariances was the best fit $(\mathrm{CFI}=0.962, \mathrm{TLI}=0.940, \mathrm{SRMR}=0.033$, RMSEA $=0.099)$. The factor loadings for the model in both samples are displayed in Table 1.

\subsubsection{Descriptive Statistics and Correlations}

Descriptive statistics and correlations among the variables are presented in Table 3. Derailment scores significantly differed by country, which were higher in the American sample than in the Japanese sample $(t=2.90, d f=950, p=0.004, d=0.19)$. Additionally, scores for present and future life satisfaction significantly differed by country, which were higher in the American sample $(t=7.22, d f=950, p<0.001, d=0.47$ for present LS; $t=7.80$, $d f=950, p<0.001, d=0.51$ for future LS). There were significant negative correlations between derailment and present and future life satisfaction in the American sample $(r=-$ $0.27, p<0.001$ for present LS; $r=-0.21, p<0.001$ for future LS), whereas the correlations in the Japanese sample were not significant or very weak $(r=-0.08$, n.s. for present $\mathrm{LS} ; r=0.09, p=0.038$ for future LS).

\subsubsection{Moderation Analyses}

As in Study 1, we conducted a series of hierarchical multiple regression analyses examining the interaction between country of the participants and derailment. The $R$-square change for both present and future life satisfaction were significant $\left(\Delta R^{2}=0.004, p=0.049\right.$ for present LS; $\Delta R^{2}=0.02, p<0.001$ for future LS). As shown in Fig. 2, simple slopes tests showed that in the American sample derailment inhibited present $(\beta=-0.22, p<0.001)$ and future life satisfaction $(\beta=-0.16, p<0.001)$, whereas derailment was not significantly related to present $(\beta=-0.09, p=0.051)$ or future life satisfaction $(\beta=0.09, p=0.061)$ in the Japanese sample. 

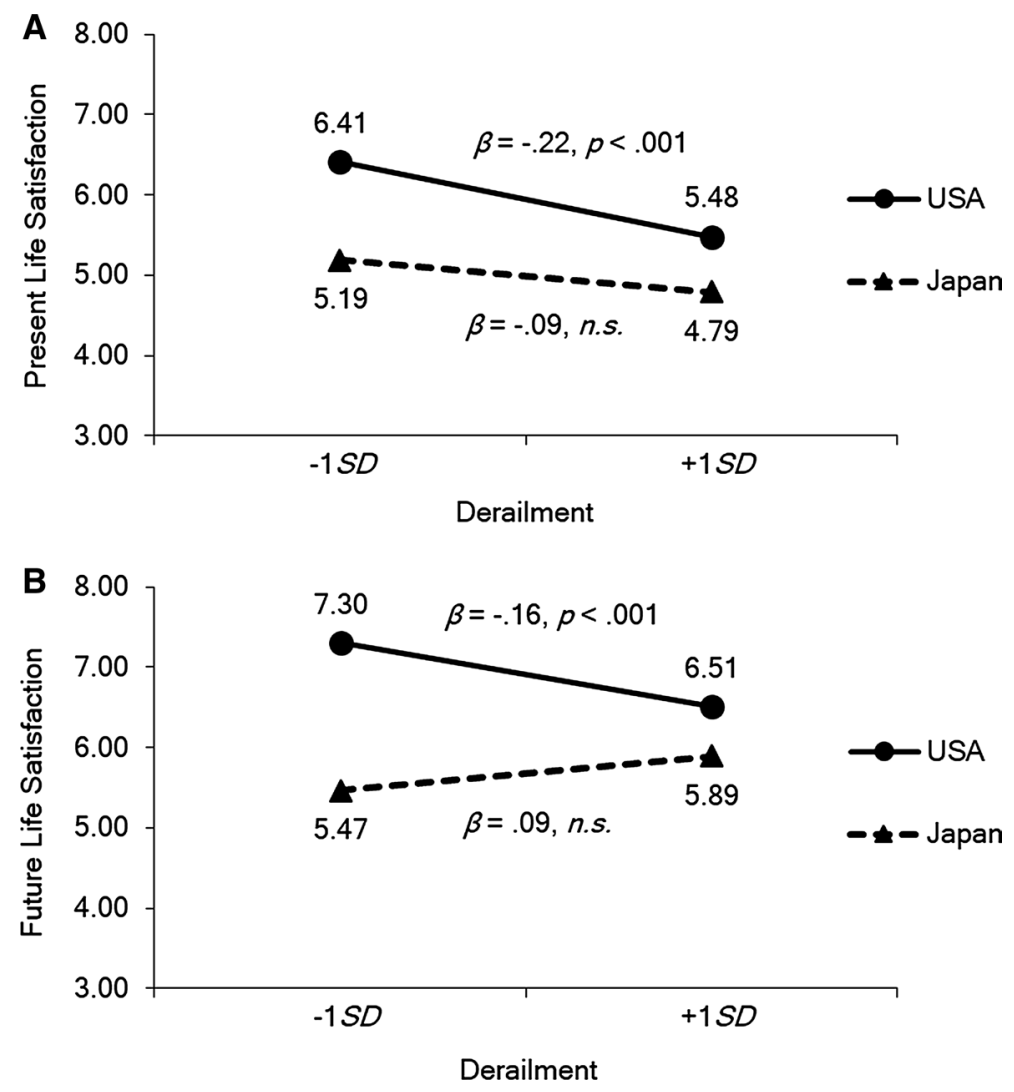

Fig. 2 Interaction Between Countries and Derailment in Study 2

\section{Study 3}

Studies 1 and 2 clearly demonstrated that derailment did not have deleterious effects on well-being in the Japanese samples. In Study 3, in accordance with Burrow et al. (2020) Study 5, we investigated whether derailment would predict subsequent well-being one year later. Well-being was assessed with measures of positive affect and depression to enhance the generalizability of the results we obtained in Studies 1 and 2.

\subsection{Method}

\subsubsection{Participants}

We recruited 1200 Japanese participants online. We excluded individuals who were under 20 years of age, did not currently live in Japan, did not pass an attention check, and did not finish the questionnaire at Time 1 or Time 2. The final sample consisted of 794 Japanese participants $\left(M_{\mathrm{age}}=54.07\right.$ years, $S D=14.88$, range $20-79 ; 48.9 \%$ were women). A 
posteriori power analysis showed that this sample size was large enough for detecting a small effect size $\left(f^{2}=0.02\right.$ resulted in $\left.1-\beta=0.90\right)$ when we ran the following linear multiple regression analyses. Employment status at Time 1 was as follows: student $=1.4 \%$, full-time employee $=35.9 \%$, part-time employee $=6.4 \%$, housewife $/$ househusband $=22.0 \%$, unemployed $=21.8 \%$, other $=12.5 \%$.

\subsubsection{Procedures}

Participants were recruited by an online survey company called Cross Marketing, which has a large sample pool in Japan. Participants were asked to answer questionnaires twice: during February to March of 2019 (Time 1) and March 2020 (Time 2) ${ }^{1}$.Participants completed online questionnaires anonymously. They consented to participation in the study before responding to the questionnaires. They were informed that their participation was voluntary without having any consequence for omitting responses or declining to participate. This study was approved by the research ethics committee of Kyoto University.

\subsubsection{Measures}

4.1.3.1 Derailment The same Derailment Scale from the two prior studies was used in Time 1. Cronbach's $\alpha=0.76$ in the current study.

4.1.3.2 Positive Affect The measures of positive affect were used in both Time 1 and 2. We assessed positive affect using the Hedonic and Eudaimonic Motives for Activities (HEMA) scale developed by Huta and Ryan (2010), after the following two modifications. First, although the original scale was developed to assess well-being "motives" (e.g., seeking enjoyment), we changed the wording of the items to assess well-being "states" (e.g., feeling enjoyment). Second, we divided affective states into two types, labelled HAP and LAP, in accordance with results using the HEMA in Asano et al. (2018) study in Japasn. It has been revealed in several studies with different samples from different countries that the original items for hedonic motives could be statistically categorized into pleasure (HAP) orientation and relaxation (LAP) orientation using factor analysis (Asano et al., 2018; Braaten et al., 2019; Bujacz et al., 2014). Based on their analysis, Asano et al. added two relaxation items, demonstrating a clear distinction between the two orientations. The items for HAP were "pleasure," "enjoyment," and "fun." The items for LAP were "relaxation," "easiness," "calmness," and "feeling laid-back." We asked participants to what extent they were currently experiencing each feeling using a response scale ranging from 1 ("not at all") to 7 ("very much"). Evidence in support of the reliability and validity of the original HEMA scale was found in an American sample (Huta \& Ryan, 2010) and Japanese sample (Asano et al., 2018). The internal consistency reliabilities of HAP and LAP in the current study were $\alpha=0.96 / 0.94$ for HAP at Time $1 / 2$ and $\alpha=0.96 / 0.95$ for LAP at Time $1 / 2$.

\footnotetext{
1 Data at Time 2 was collected March 11-18, 2020. Although the first COVID-19 positive case in Japan was confirmed in January, the number of new cases were not large until the end of March, counting under 100 cases per day. Indeed, our data showed the mean level of depression at T2 was lower than at T1 $\left(M_{\mathrm{T} 1}\right.$ $=0.86, M_{\mathrm{T} 2}=0.63, p<.000, d=.28$. see the results section). Also, mean levels of HAP and LAP were not significantly different between T1 and T2. Based on the situation in Japan, timing of collection of data, and these results, we did not have any evidence to indicate that the results in Study 3 were affected by the outbreak of COVID-19
} 
Table 4 Descriptive statistics and correlation matrix in study 3

\begin{tabular}{|c|c|c|c|c|c|c|c|c|}
\hline & $M$ & $S D$ & 1 & 2 & 3 & 4 & 5 & 6 \\
\hline 1. Derailment & 3.04 & 0.65 & - & & & & & \\
\hline 2. HAP (Time 1) & 4.53 & 1.30 & $-.14 * * *$ & - & & & & \\
\hline 3. LAP (Time 1) & 4.55 & 1.31 & $-.18 * * *$ & $.60 * * *$ & - & & & \\
\hline 4. Depression (Time 1) & 0.86 & 0.88 & $.25 * * *$ & $-.56 * * *$ & $-.56^{* * *}$ & - & & \\
\hline 5. HAP (Time 2) & 4.48 & 1.29 & $-.17 * * *$ & $.65 * * *$ & $.45^{* * *} *$ & $-.48 * * *$ & - & \\
\hline 6. LAP (Time 2) & 4.53 & 1.35 & $-.14 * * *$ & $.45 * * *$ & $.64 * * *$ & $-.47 * * *$ & $.66 * * *$ & - \\
\hline 7. Depression (Time 2) & 0.63 & 0.78 & $.19 * * *$ & $-.45 * * *$ & $-.44 * * *$ & $.71 * * *$ & $-.49 * * *$ & $-.50 * * *$ \\
\hline
\end{tabular}

$\mathrm{HAP}=$ high-arousal positive affect, LAP $=$ low-arousal positive affect $* * * p<.001$

4.1.3.3 Depression Depression was assessed at Time 1 and Time 2 using the 6-item Kessler Psychological Distress Scale (K6; Kessler et al., 2003). The scale asks participants how frequently they have experienced each of six symptoms of major depression and generalized anxiety disorder during the past month. Example items include, "How often did you feel hopeless?" and "How often did you feel so depressed that nothing could cheer you up?" Items are rated on a 5-point Likert scale ranging from 0 ("none of the time") to 4 ("all of the time"). Evidence in support of the reliability and validity of the K6 was found in an American sample (Kessler et al., 2003) and Japanese sample (Furukawa et al., 2008). The alpha coefficients in the current study were 0.91 at both Time 1 and Time 2.

\subsection{Results}

\subsubsection{Measurement Tests of the Derailment Scale}

Following the same method of Studies 1 and 2, we performed PCA and CFA, demonstrating the unidimentionality (Eigenvalues were $2.801,0.942,0.814,0.619 \ldots$ ) and good enough fit indices for the modified model $(\mathrm{CFI}=0.983$, TLI $=0.964$, SRMR $=0.021$, RMSEA $=0.060$ ).

\subsubsection{Descriptive Statistics and Correlations}

Descriptive statistics and correlations among the variables are presented in Table 4. As a preliminary analysis, we tested whether scores were different between Time 1 and Time 2 using paired $t$-tests. Results showed that the differences in HAP and LAP scores between Times 1 and 2 were not significant $(t=1.38, d f=793, p=0.169, d=0.04$ for HAP; $t=0.53$, $d f=793, p=0.594, d=0.02$ for LAP). Depression scores significantly decreased over time $(t=10.20, d f=793, p<0.001, d=0.28)$. Correlations between derailment and HAP and LAP at Time 1 were modestly negative $(r=-0.14, p<0.001$ for HAP; $r=-0.18$, $p<0.001$ for LAP). In addition, derailment significantly positively correlated with depression at Time $1(r=0.25, p<0.001)$. 
Table 5 Multiple regression analyses in study 3

\begin{tabular}{llllll}
\hline & $B$ & $95 \% C I$ & $t$ & $\beta$ & $p$ \\
\hline DV: HAP (Time 2) & & & & & \\
Age & .008 & {$[.004 .013]$} & 3.49 & .094 & .001 \\
Gender (1= male, 2=female) & .038 & {$[-.099 .174]$} & 0.54 & .015 & .588 \\
HAP (Time 1) & .622 & {$[.568 .675]$} & 22.93 & .627 & $<.000$ \\
Derailment & -.131 & {$[-.237-.026]$} & -2.44 & -.066 & .015 \\
DV: LAP (Time 2) & & & & .169 & $<.000$ \\
Age & .015 & {$[.010 .020]$} & 6.04 & .046 & .090 \\
Gender (1=male, 2=female) & .123 & {$[-.019 .265]$} & 1.70 & .046 & $<.000$ \\
LAP (Time 1) & .602 & {$[.545 .659]$} & 20.68 & .584 & .367 \\
Derailment & -.051 & {$[-.162 .060]$} & -0.90 & -.025 & $<.000$ \\
DV: Depression (Time 2) & & & & & .592 \\
Age & -.005 & {$[-.007-.002]$} & -3.52 & -.092 & $<.000$ \\
Gender (1=male, 2=female) & .021 & {$[-.056 .098]$} & 0.54 & .013 & .803 \\
Depression (Time 1) & .600 & {$[.554 .647]$} & 25.45 & .679 & .006 \\
Derailment & .008 & {$[-.053 .069]$} & 0.25 & & \\
\hline
\end{tabular}

$\mathrm{DV}=$ dependent variable, HAP $=$ high-arousal positive affect, LAP = low-arousal positive affect, CI $=$ confidence interval

\subsubsection{Effects of Derailment on Well-Being}

We conducted a series of multiple regression analyses examining the effects of derailment at Time 1 on well-being in Time 2. Age, gender, each well-being variable at Time 1 (HAP, LAP, and depression), and derailment were included as independent variables. The dependent variables were the three well-being variables at Time 2. As shown in Table 5, derailment at Time 1 showed a significant negative relationship with HAP at Time 2, although the coefficient was very small $(\beta=-0.066, p=0.015)$. Derailment had no significant effect on $\operatorname{LAP}(\beta=-0.025, p=0.367)$ or depression $(\beta=0.006, p=0.803)$ at Time 2 .

\section{Discussion}

Current research with East Asian samples challenged the existing postulation that derailment is detrimental to mental health (Burrow et al., 2020; Ratner et al., 2019, 2020). Previous studies had not examined the effects of derailment in non-WEIRD (White, Educated, Industrialized, Rich, and Democratic) samples (Henrich et al., 2010). The results of the current studies in which several different measures of well-being were utilized with Japanese samples revealed that the harmful effect of derailment was very subtle in most cases. These findings contribute to our understanding of identity or well-being in cultural context, because no studies had examined how culture plays a role in the effect of derailment on well-being. Below, we discuss these findings and the implications of these results for research in cultural psychology, as well as the limitations.

As predicted in Hypothesis 1, derailment in East Asians had a weaker negative correlation with well-being than in North Americans. In Study 1, derailment was negatively 
related to present and future life satisfaction in the Canadian undergraduate sample, whereas in the Japanese undergraduate sample derailment was not related to life satisfaction. Study 2 replicated and expanded these results using adult samples in the United States and in Japan with different measures of life satisfaction; in particular, future life satisfaction referred to a particular time span (10 years into the future). These results are in line with the findings in previous studies about the relationship between instability of the selfconcept and well-being across cultures (Church et al., 2014; Haas \& van Dellen, 2020).

We also found support for Hypothesis 2, which was that East Asians who feel derailed would not experience long-term damage to their well-being. In the Japanese sample of Study 3, there was no effect of derailment on depressive symptoms or relaxation orientation (LAP) one year later, although there was a weak but significant relationship to subsequent pleasure orientation (HAP). Nevertheless, our findings are dissimilar to the results of Burrow et al. (2020) in their sample of American undergraduate students. Burrow et al. found that derailment at Time 1 predicted depressive symptoms 18 months later. However, we cannot rule out the possibility that the difference in the findings between the two samples is due to age-related differences as opposed to cultural differences between the two countries, because the participants in Burrow et al.'s study were students, whereas the present study involved a sample of adults. Future research is needed to clarify the different influences of age and culture on the effects of derailment.

Despite the fact that our studies provide no data that directly answer the question of why these differences were found in the two cultural groups, we can speculate based on prior research. East Asians have a belief that one's life trajectory across the lifespan is not linear but fluctuating or cyclical (Ji et al., 2001), helping them reappraise a negative trend in their life into one that has a promising outlook. Likewise, they have a unique propensity, called dialecticism, to accept complex inconsistencies of self-concepts (Heine, 2001; Peng \& Nisbett, 1999; Spencer-Rodgers et al., 2010). As such, East Asians compared to North Americans believe that their identity is something changeable corresponding to contexts, situations, or temporal timings. Previous studies showed that the association between culture and cross-situational (not temporal) inconsistency of the self-concept was fully mediated by dialecticism (Church et al., 2012; English \& Chen, 2007; Spencer-Rodgers et al., 2009). Thus, it can be argued that this holistic, changeable, and contradictory nature of self or life in East Asians let people accept their fluctuation of the self and plays a role in inhibiting the deleterious influence of feeling derailed. Further research could directly assess these culture-specific beliefs to test these assumptions, which would provide more robust evidence of the influence of cultural factors and make what underlies the cultural differences more comprehensible.

\subsection{Implications and Impact}

This research study makes several contributions and offers some insights. First, we confirmed that the Japanese version of the Derailment Scale was compatible with the original version using invariance tests. According to Boer et al. (2018), metric invariance is desirable, whereas scalar invariance is not a must, for comparing associations of variables across cultural contexts. However, it is noteworthy that the factor loadings of Item 2 in the Japanese samples were more modest than the other items, especially in Studies 1 and 3 . This could be explained by the different perception of connectedness among temporally expanded selves. Previous studies showed that East Asians were more likely to feel connected to their past self than Westerners (Ji et al., 2019), which was also found in the 
current study. ${ }^{2}$ Lower factor loadings on this item indicate that Japanese, compared to Canadians and Americans, may have the belief that having a different self-concept from the past self does not necessarily imply a lack of connection with the past self; that is, they may be better at reconciling the pathway through which previously held identities have become current ones, even if they recognize that they have changed very much over time. This interpretation is consistent with a holistic view of the life course and with the dialectical thinking characteristic of accepting contradictory situations (Nisbett et al., 2001; Peng \& Nisbett, 1999; Spencer-Rodgers et al. 2009, 2010).

Additionally, as Spencer-Rodgers (2010) mentioned in their review, despite a large amount of cross-cultural studies published about relational (cross-situational or cross-role) instability of the self-concept in social contexts (e.g., Cross et al., 2003; English \& Chen, 2007, 2011; Oishi et al., 2004; Suh, 2002), the number of studies on temporally expanded self-concept instability was considerably limited (Chopik \& Kitayama, 2018; Church et al., 2012, 2014; Haas \& van Dellen, 2020). Our findings accentuate that cultural differences can be observed not only in relational but also in temporal instability of identity, because East Asians have a unique propensity to accept the fluctuations in self-concept based on their dialectical perspective.

\subsection{Limitations and Future Directions}

The current studies have several limitations that deserve mentioning. First, all participants in Study 3 were Japanese, thereby lacking a comparison with a Western sample. To directly highlight the underlying cultural effects, future research needs to include comparison samples from other countries. Although the comparison between North Americans and East Asians is a worthy contrast of Western and Eastern cultures, investigating derailment and its effects in other countries would shed light on cultural variation in the sensitivity and consequences of derailment. This approach would also contribute to identifying whether the lower significance of derailment and its effects are particular to Japan.

Second, although we assessed low-arousal positive affect as culture-specific well-being, future research can include not only hedonic well-being such as life satisfaction and positive affect, but also eudaimonic well-being such as purpose in life, autonomy, and personal growth (Ryff \& Keyes, 1995). Similarly, including interdependent happiness (Hitokoto \& Uchida, 2015), which is harmony with others, quiescence, and ordinariness, would be intriguing and valuable because it is known to be prominent in East Asian cultures.

Third, it may be that one's understanding of the concept of derailment may differ among individuals. One concern is that the valence of change differs among individuals. When reading and responding to the items, the wording does not suggest improvement (positive change) or decline (negative change), rather the items maintain an ambiguous nuance. Thus, the interpretation of items all depends on the respondents. Burrow et al. (2020) pointed out and tackled this point, displaying that the correlation between derailment and positive valence of subjective change in self-concept was

\footnotetext{
2 The score of the Japanese participants on Item 2, "I do not feel very connected to who I was in the past," was lower than the score of the Canadian and American participants across studies. In Study 1, the difference between Japanese scores $(M=2.49)$ and Canadian scores $(M=3.01)$ were significant $(t=10.33, d f$ $=1,737, p<.000, d=.50)$. In Study 2, the difference between Japanese scores $(M=2.48)$ and American scores $(M=2.66)$ were also significant $(t=2.49, d f=950, p=.013, d=.16)$.
} 
slightly negative. This implies that the participants recognized the change expressed in the Derailment Scale as modestly negative, whereas this is not clear in East Asian participants. Thus, future research may distinguish different directions of change (positive, negative, neutral, or even non-linear) in the self and life direction, designing experimental manipulations so that the influence of direction of change would be comprehensible. Another concern is that we do not know whether East Asians rather than North Americans perceive derailment as less important in their life path and feel more comfortable with their inconsistency of the self, because participants were not specifically asked this question. Therefore, future studies may wish to directly assess how individuals interpret the inconsistency, and how the interpretation differs across cultures, using both quantitative and qualitative methods.

Lastly, in the measurement of derailment, it is not specified what the feelings of being derailed are due to. Therefore, the results are limited in understanding what types of transitions or experiences are associated with derailment. For example, there may be differences in derailment when the life-changing event is more traumatic (e.g., loss of a job, experiencing a heart attack) versus being a more neutral event (e.g., changing a college major, turning 40 years old). Although Burrow et al. (2020) provided evidence that experiencing an objective event was unrelated to feeling derailed, implying the perception of derailment is independent of the occurrence of life-changing events, the participants in their studies were all North Americans. Future research could clarify whether the type of transition or life-changing event associated with derailment contributes to differences in derailment across cultures.

\section{Conclusion}

Individuals often experience difficulty in accepting the difference between one's past and present identities, and the consistent changes in life direction. This subjective experience diminishes their positive affect and fosters depression. The current research examined how the feeling of derailment and its psychological consequences differs culturally by targeting North American and Japanese samples. Two cross-sectional studies and one longitudinal study demonstrated that the detrimental effect of derailment was not observed in the Japanese samples unlike the North American samples. These cultural contrasts could be derived from the East Asian dialectical beliefs that life or the self can be permanently changing in different directions. Thus, Japanese people are more likely to accept inconsistency without conflict, whether it is between changes in identity throughout their lives or in ones' life direction itself.

Supplementary Information The online version contains supplementary material available at (https://doi. org/10.1007/s10902-021-00375-4).

Funding This research was financially supported by grants from the Japanese Society for the Promotion of Science [grant number 18J01883].

Data availability The data and code that support the findings of this research are available on request from the corresponding author. The data are not publicly available due to privacy or ethical restrictions. 


\section{Declarations}

Conflict of interest The authors declare there are no potential conflicts of interest with respect to the research, authorship, and/or publication of this article.

\section{References}

Aiken, L. S., \& West, S. G. (1991). Multiple regression: Testing and interpreting interactions. USA: Sage Publications Inc.

Asano, R., Tsukamoto, S., Igarashi, T., \& Huta, V. (2018). Psychometric properties of measures of hedonic and eudaimonic orientations in Japan: The HEMA scale. Current Psychology. https://doi.org/10.1007/ s12144-018-9954-Z

Ball, L., \& Chandler, M. (1989). Identity formation in suicidal and nonsuicidal youth: The role of self-continuity. Development and Psychopathology, 1(3), 257-275. https://doi.org/10.1017/S0954579400000444

Boer, D., Hanke, K., \& He, J. (2018). On detecting systematic measurement error in cross-cultural research: A review and critical reflection on equivalence and invariance tests. Journal of Cross-Cultural Psychology, 49(5), 713-734. https://doi.org/10.1177/0022022117749042

Boucher, H. C. (2011). The dialectical self-concept II: Cross-role and within-role consistency, well-being, self-certainty, and authenticity. Journal of Cross-Cultural Psychology, 42(7), 1251-1271. https://doi. org/10.1177/0022022110383316

Braaten, A., Huta, V., Tyrany, L., \& Thompson, A. (2019). Hedonic and eudaimonic motives toward university studies: How they relate to each other and to well-being derived from school. Journal of Positive Psychology and Wellbeing, 3(2), 179-196.

Brislin, R. W. (1986). The wording of translation of research instruments. In W. J. Lonner \& J. W. Berry (Eds.), Field methods in cross-cultural research (pp. 137-164). New York: Sage.

Bujacz, A., Vittersø, J., Huta, V., \& Kaczmarek, L. D. (2014). Measuring hedonia and eudaimonia as motives for activities: Cross-national investigation through traditional and Bayesian structural equation modeling. Frontiers in Psychology, 5, 984. https://doi.org/10.3389/fpsyg.2014.00984

Burrow, A. L., Hill, P. L., Ratner, K., \& Fuller-Rowell, T. E. (2020). Derailment: Conceptualization, measurement, and adjustment correlates of perceived change in self and direction. Journal of Personality and Social Psychology, 118(3), 584-601. https://doi.org/10.1037/pspp0000209

Burrow, A. L., Sumner, R., \& Ong, A. D. (2014). Perceived change in life satisfaction and daily negative affect: The moderating role of purpose in life. Journal of Happiness Studies, 15(3), 579-592. https:// doi.org/10.1007/s10902-013-9436-9

Byrne, B. M. (2013). Structural equation modeling with Mplus: Basic concepts, applications, and programming. Routledge.

Campbell, J. D., Trapnell, P. D., Heine, S. J., Katz, I. M., Lavallee, L. F., \& Lehman, D. R. (1996). Selfconcept clarity: Measurement, personality correlates, and cultural boundaries. Journal of Personality and Social Psychology, 70(1), 141-156. https://doi.org/10.1037/0022-3514.70.1.141

Chishima, Y., Murakami, T., Worrell, F. C., \& Mello, Z. R. (2019). The Japanese version of the Adolescent Time Inventory-Time Attitudes (ATI-TA) scale: Internal consistency, structural validity, and convergent validity. Assessment, 26(2), 181-192. https://doi.org/10.1177/1073191116683800

Chopik, W. J., \& Kitayama, S. (2018). Personality change across the life span: Insights from a cross-cultural, longitudinal study. Journal of Personality, 86(3), 508-521. https://doi.org/10.1111/jopy.12332

Church, A. T., Alvarez, J. M., Katigbak, M. S., Mastor, K. A., Cabrera, H. F., Tanaka-Matsumi, J., de Jesús Vargas-Flores, J., Ibáñez-Reyes, J., Zhang, H., Shen, J., Locke, K. D., Ortiz, F. A., Curtis, G. J., Simon, J.-Y.R., Ching, C. M., \& Buchanan, A. L. (2012). Self-concept consistency and short-term stability in eight cultures. Journal of Research in Personality, 46(5), 556-570. https://doi.org/10.1016/j.jrp.2012.06.003

Church, A. T., Katigbak, M. S., Ibáñez-Reyes, J., de Jesús Vargas-Flores, J., Curtis, G. J., Tanaka-Matsumi, J., Cabrera, H. F., Mastor, K. A., Zhang, H., Shen, J., Locke, K. D., Alvarez, J. M., Ching, C. M., Ortiz, F. A., \& Simon, J.Y.R. (2014). Relating self-concept consistency to hedonic and eudaimonic well-being in eight cultures. Journal of Cross-Cultural Psychology, 45(5), 695-712. https://doi.org/10.1177/0022022114527347

Cross, S. E., Gore, J. S., \& Morris, M. L. (2003). The Relational-interdependent self-construal, self-concept consistency, and well-being. Journal of Personality and Social Psychology, 85(5), 933-944. https://doi. org/10.1037/0022-3514.85.5.933

Diener, E. (1984). Subjective well-being. Psychological Bulletin, 95(3), 542-575. https://doi. org/10.1037/0033-2909.95.3.542 
Ding, L., Velicer, W. F., \& Harlow, L. L. (1995). Effects of estimation methods, number of indicators per factor, and improper solutions on structural equation modeling fit indices. Structural Equation Modeling, 2(2), 119-143. https://doi.org/10.1080/10705519509540000

English, T., \& Chen, S. (2007). Culture and self-concept stability: Consistency across and within contexts among Asian Americans and European Americans. Journal of Personality and Social Psychology, 93(3), 478-490. https://doi.org/10.1037/0022-3514.93.3.478

English, T., \& Chen, S. (2011). Self-concept consistency and culture: The differential impact of two forms of consistency. Personality and Social Psychology Bulletin, 37(6), 838-849. https://doi.org/10.1177/0146167211 400621

Erikson, E. H. (1968). Identity: Youth and crisis. WW Norton \& Company.

Furukawa, T. A., Kawakami, N., Saitoh, M., Ono, Y., Nakane, Y., Nakamura, Y., Tachimori, H., Iwata, N., Uda, H., Nakane, H., Watanabe, M., Naganuma, Y., Hata, Y., Kobayashi, M., Miyake, Y., Takeshima, T., \& Kikkawa, T. (2008). The performance of the Japanese version of the K6 and K10 in the World Mental Health Survey Japan. International Journal of Methods in Psychiatric Research, 17(3), 152-158. https://doi.org/10.1002/ mpr.257

Haas, B. W., \& van Dellen, M. R. (2020). Culture is associated with the experience of long-term self-concept changes. Advance online publication. https://doi.org/10.1177/1948550619893966

Harter, S., Bresnick, S., Bouchey, H. A., \& Whitesell, N. R. (1997). The development of multiple role-related selves during adolescence. Development and Psychopathology, 9(4), 835-853. https://doi.org/10.1017/ S0954579497001466

Hayes, A. F. (2013). Introduction to mediation, moderation, and conditional process analysis: A regressionbased approach. New york: Guilford Press.

Heine, S. J. (2001). Self as cultural product: An examination of East Asian and North American selves. Journal of Personality, 69(6), 881-906. https://doi.org/10.1111/1467-6494.696168

Henrich, J., Heine, S. J., \& Norenzayan, A. (2010). The weirdest people in the world? Behavioral and Brain Sciences, 33(2-3), 61-83. https://doi.org/10.1017/S0140525X0999152X

Hitokoto, H., \& Uchida, Y. (2015). Interdependent happiness: Theoretical importance and measurement validity. Journal of Happiness Studies, 16(1), 211-239. https://doi.org/10.1007/s10902-014-9505-8

Huta, V. (2016). Eudaimonic and hedonic orientations: Theoreticalconsiderations and research findings. In J. Vitters $\emptyset$ (Ed.), Handbook of eudaimonic well-being (pp. 215-231). Springer: New york.

Huta, V., \& Ryan, R. M. (2010). Pursuing pleasure or virtue: The differential and overlapping well-being benefits of hedonic and eudaimonic motives. Journal of Happiness Studies, 11(6), 735-762. https://doi. org/10.1007/s10902-009-9171-4

Ji, L. J., Hong, E. K., Guo, T., Zhang, Z., Su, Y., \& Li, Y. (2019). Culture, psychological proximity to the past and future, and self-continuity. European Journal of Social Psychology, 49(4), 735-747. https://doi. org/10.1002/ejsp. 2544

Ji, L. J., Nisbett, R. E., \& Su, Y. (2001). Culture, change, and prediction. Psychological Science, 12(6), 450456. https://doi.org/10.1111/1467-9280.00384

Kessler, R. C., Barker, P. R., Colpe, L. J., Epstein, J. F., Gfroerer, J. C., Hiripi, E., Howes, M. J., Normand, S.L.T., Manderscheid, R. W., Walters, E. E., \& Zaslavsky, A. M. (2003). Screening for serious mental illness in the general population. Archives of General Psychiatry, 60(2), 184-189. https://doi.org/10.1001/archp syc.60.2.184

Keyes, C. L. M. (2000). Subjective change and its consequences for emotional well-being. Motivation and Emotion, 24(2), 67-84. https://doi.org/10.1023/A:1005659114155

Keyes, C. L. M., \& Ryff, C. D. (2000). Subjective change and mental health: A self-concept theory. Social Psychology Quarterly, 63(3), 264-279. https://doi.org/10.2307/2695873

Lachman, M. E., Röcke, C., Rosnick, C., \& Ryff, C. D. (2008). Realism and illusion in Americans' temporal views of their life satisfaction: Age differences in reconstructing the past and anticipating the future. Psychological Science, 19(9), 889-897. https://doi.org/10.1111/j.1467-9280.2008.02173.x

Marsh, H. W., Hau, K.-T., \& Wen, Z. (2004). In search of golden rules: Comment on hypothesis-testing approaches to setting cutoff values for fit indexes and dangers in overgeneralizing Hu and Bentler's (1999) findings. Structural Equation Modeling, 11(3), 320-341. https://doi.org/10.1207/s15328007sem1103_2

McAdams, D. P., \& McLean, K. C. (2013). Narrative identity. Current Directions in Psychological Science, 22(3), 233-238. https://doi.org/10.1177/0963721413475622

Meeus, W., Iedema, J., Helsen, M., \& Vollebergh, W. (1999). Patterns of adolescent identity development: Review of literature and longitudinal analysis. Developmental Review, 19(4), 419-461. https://doi. org/10.1006/drev.1999.0483

Mello, Z. R., Zhang, J. W., Barber, S. J., Paoloni, V. C., Howell, R. T., \& Worrell, F. C. (2016). Psychometric properties of time attitude scores in young, middle, and older adult samples. Personality and Individual Differences, 101, 57-61. https://doi.org/10.1016/j.paid.2016.05.037 
Nisbett, R. E., Peng, K., Choi, I., \& Norenzayan, A. (2001). Culture and systems of thought: Holistic versus analytic cognition. Psychological Review, 108(2), 291-310. https://doi.org/10.1037/0033-295X.108.2.291

Oishi, S., Diener, E., Napa Scollon, C., \& Biswas-Diener, R. (2004). Cross-situational consistency of affective experiences across cultures. Journal of Personality and Social Psychology, 86(3), 460-472. https://doi. org/10.1037/0022-3514.86.3.460

Peng, K., \& Nisbett, R. E. (1999). Culture, dialectics, and reasoning about contradiction. American Psychologist, 54(9), 741-754. https://doi.org/10.1037/0003-066X.54.9.741

Ratner, K., Burrow, A. L., \& Mendle, J. (2020). The unique predictive value of discrete depressive symptoms on derailment. Journal of Affective Disorders, 270, 65-68. Advance Online Publication.

Ratner, K., Mendle, J., Burrow, A. L., \& Thoemmes, F. (2019). Depression and derailment: A cyclical model of mental illness and perceived identity change. Clinical Psychological Sciences, 7(4), 735-753. https://doi. org/10.1177/2167702619829748

Roberts, B. W., Walton, K. E., \& Viechtbauer, W. (2006). Patterns of mean-level change in personality traits across the life course: A meta-analysis of longitudinal studies. Psychological Bulletin, 132(1), 1-25. https ://doi.org/10.1037/0033-2909.132.1.1

Robins, R. W., Noftle, E. E., Trzesniewski, K. H., \& Roberts, B. W. (2005). Do people know how their personality has changed? Correlates of perceived and actual personality change in young adulthood. Journal of Personality, 73(2), 489-521. https://doi.org/10.1111/j.1467-6494.2005.00317.x

Ryan, R. M., \& Deci, E. L. (2001). On happiness and human potentials: A review of research on hedonic and eudaimonic well-being. Annual Review of Psychology, 52(1), 141-166. https://doi.org/10.1146/annur ev.psych.52.1.141

Ryff, C. D., \& Keyes, C. L. M. (1995). The structure of psychological well-being revisited. Journal of Personality and Social Psychology, 69(4), 719-727. https://doi.org/10.1037/0022-3514.69.4.719

Schimmack, U. (2008). The structure of subjective well-being. In M. Eid \& M. Larsen (Eds.), The science of subjective well-being (pp. 97-123). New York: Guilford Press.

Sedikides, C., Wildschut, T., Routledge, C., \& Arndt, J. (2015). Nostalgia counteracts self-discontinuity and restores self-continuity. European Journal of Social Psychology, 45(1), 52-61. https://doi.org/10.1002/ ejsp. 2073

Specht, J., Egloff, B., \& Schmukle, S. C. (2011). Stability and change of personality across the life course: The impact of age and major life events on mean-level and rank-order stability of the big five. Journal of Personality and Social Psychology, 101(4), 862-882. https://doi.org/10.1037/a0024950

Spencer-Rodgers, J., Boucher, H. C., Mori, S. C., Wang, L., \& Peng, K. (2009). The dialectical self-concept: Contradiction, change, and holism in East Asian cultures. Personality and Social Psychology Bulletin, 35(1), 29-44. https://doi.org/10.1177/0146167208325772

Spencer-Rodgers, J., Williams, M. J., \& Peng, K. (2010). Cultural differences in expectations of change and tolerance for contradiction: A decade of empirical research. Personality and Social Psychology Review, 14(3), 296-312. https://doi.org/10.1177/1088868310362982

Suh, E. M. (2002). Culture, identity consistency, and subjective well-being. Journal of Personality and Social Psychology, 83(6), 1378-1391. https://doi.org/10.1037/0022-3514.83.6.1378

Tsai, J. L., Knutson, B., \& Fung, H. H. (2006). Cultural variation in affect valuation. Journal of Personality and Social Psychology, 90(2), 288-307. https://doi.org/10.1037/0022-3514.90.2.288

Wilson, A. E., \& Ross, M. (2001). From chump to champ: People's appraisals of their earlier and present selves. Journal of Personality and Social Psychology, 80(4), 572-584. https://doi. org/10.1037/0022-3514.80.4.572

Worrell, F. C., Mello, Z. R., \& Buhl, M. (2013). Introducing English and German versions of the adolescent time attitude scale. Assessment, 20(4), 496-510. https://doi.org/10.1177/1073191110396202

Publisher's Note Springer Nature remains neutral with regard to jurisdictional claims in published maps and institutional affiliations. 